\title{
Mechanism of Ferroptosis and Its Role in Type 2 Diabetes Mellitus
}

\author{
Wenxin Sha $\mathbb{D}^{1}$, Fei Hu $\mathbb{D}^{1,2}$ Yang Xi $\mathbb{D}^{1},{ }^{1}$ Yudong Chu $\mathbb{D}^{1,3}$ and Shizhong Bu $\mathbb{D}^{1}$ \\ ${ }^{1}$ Diabetes Research Center, School of Medicine, Ningbo University, Ningbo 315211, China \\ ${ }^{2}$ Cixi Biomedical Research Institute, Wenzhou Medical University, Cixi 315300, China \\ ${ }^{3}$ Department of Nephrology, Ningbo Medical Center Lihuili Hospital, Ningbo 315100, China \\ Correspondence should be addressed to Yudong Chu; chuyudong1982@163.com and Shizhong Bu; bushizhong@nbu.edu.cn
}

Received 24 March 2021; Accepted 21 June 2021; Published 28 June 2021

Academic Editor: Almudena G mez Hern ndez

Copyright (c) 2021 Wenxin Sha et al. This is an open access article distributed under the Creative Commons Attribution License, which permits unrestricted use, distribution, and reproduction in any medium, provided the original work is properly cited.

\begin{abstract}
Ferroptosis is a novel form of nonapoptotic regulated cell death (RCD). It features iron-dependent lipid peroxide accumulation accompanied by inadequate redox enzymes, especially glutathione peroxidase 4 (GPX4). RAS-selective lethal 3 (RSL3), erastin, and ferroptosis inducing 56 (FIN56) induce ferroptosis via different manners targeting GPX4 function. Acyl-CoA synthetase long-chain family 4 (ACSL4), lysophosphatidylcholine acyltransferase 3 (LPCAT3), and lipoxygenases (LOXs) participate in the production of lipid peroxides. Heat shock protein family B member 1 (HSPB1) and nuclear receptor coactivator 4 (NCOA4) regulate iron homeostasis preventing ferroptosis caused by the high concentration of intracellular iron. Ferroptosis is ubiquitous in our body as it exists in both physiologic and pathogenic processes. It is involved in glucose-stimulated insulin secretion (GSIS) impairment and arsenic-induced pancreatic damage in the pathogenesis of diabetes. Moreover, iron and the iron-sulfur (Fe-S) cluster influence each other, causing mitochondrial iron accumulation, more reactive oxygen species (ROS) production, endoplasmic reticulum (ER) stress, failure in biosynthesis of insulin, and ferroptosis in $\beta$-cells. In addition, ferroptosis also engages in the pathogenesis of diabetic complications such as myocardial ischemia and diabetic cardiomyopathy (DCM). In this review, we summarize the mechanism of ferroptosis and especially its association with type 2 diabetes mellitus (T2DM).
\end{abstract}

\section{Introduction}

Ferroptosis, which was first defined in 2012, is a form of nonapoptotic regulated cell death (RCD) because it takes place without caspases, a family of cysteine proteases cleaving specific intracellular substrates leading to apoptosis [1-3]. In other words, ferroptosis occurs without the key effectors of apoptosis such as BAX, BAK, and caspases [4]. Notably, ferroptosis is dependent on intracellular iron instead of any other metals, and it is also morphologically and biochemically different from other types of RCD such as receptorinteracting protein kinase 1- (RIPK1-) dependent necroptosis (a regulated form of necrosis) and apoptosis-inducing factor 1 -dependent parthanatos $[5,6]$. It also does not involve the key factors of necroptosis such as MLKL, RIPK1, and RIPK3 [7].

Ferroptosis features intracellular iron overloaded and iron-dependent lipid peroxide accumulation. Additionally, ferroptosis also leads to a suppression of oxidoreductase especially glutathione peroxidase 4 (GPX4), a lipid peroxide scavenger [8]. Ferroptosis inhibitors rescue GPX4-deficient $\mathrm{T}$ cells from dying of lipid peroxide accumulation-induced ferroptosis [9]. Moreover, vitamin E can compensate for the lack of GPX4 as an antioxidant while vitamin C cannot probably due to its water-soluble property [9]. In some cells such as kidney cells, ferroptosis is accompanied by impaired mitochondria with reduced organelle size, disappearance of mitochondrial cristae, and rupture of the mitochondrial outer membrane [10].

Ferroptosis has been studied in many pathogenic processes since it was identified [11]. Many experiments are designed to investigate diseases related to neurons (e.g., Parkinson's disease), immune cells (e.g., diffuse B cell lymphoma), and kidney cells (e.g., acute kidney injuries) [11-14].

Ferroptosis inhibitors were found to prevent cell death better than other inhibitors such as autophagy inhibitor (3methyladenine), necroptosis inhibitor (necrostatin-1), and apoptosis inhibitor (pan-caspase inhibitor z-VAD-fmk) [9, 
15, 16]. Ferroptosis inhibitors preserved mitochondrial membrane potential $(\Delta \Psi \mathrm{m})$, decreased lipid peroxidation, and reduced nonheme iron in mitochondria [15]. Furthermore, significantly lowered levels of cardiac nonheme iron and myocardial enzymes during ischemia-reperfusion injury (IRI) by ferroptosis inhibitors were observed in a DOX-induced cardiotoxicity rodent model [15]. Moreover, immune-cell infiltration into postischemic area was reduced in acute kidney injuries [17].

The role of ferroptosis in tumor development and proliferation is under intensive investigation. Some studies find that ferroptosis facilitates oncogenesis $[18,19]$, while other studies report inhibition of tumor cell proliferation by ferroptosis [20, 21]. An earlier study demonstrated that P53 promotes ferroptosis through inhibiting the expression of solute carrier family 7 member 11 (SLC7A11), a key component of the cystine/glutamate antiporter $\left(x_{c}{ }^{-}\right)$system [22]. Another transcriptional target of P53, spermidine/spermine N1-acetyltransferase 1 (SAT1), was found to promote ferroptosis as well but through elevating the expression of arachidonate 15-lipoxygenase [23]. However, not all the transcriptional targets of P53 are promoters of ferroptosis. For example, the glutaminase 2 (GLS2, a transcriptional target of P53) can function as an antioxidant, which makes it potentially an inhibitor of ferroptosis [24]. Furthermore, the knockdown of GLS2 inhibits ferroptosis in fibroblasts [25]. Moreover, P53 can suppress ferroptosis through the DPP4-dependent pathway [26].

\section{Mechanism of Ferroptosis}

Intracellular iron overloaded and excessive lipid peroxides are considered the lethal elements to trigger ferroptosis [8]. The accumulation of polyunsaturated fatty acids (e.g., arachidonoyl) and reduced lipid peroxide scavenging such as the inhibition of lipid antioxidants (e.g., GPX4) lead to ferroptosis. A general review of the mechanism of ferroptosis and its association with other forms of cell death is discussed below.

\subsection{Lipid Peroxide Production. Arachidonoyl- (AA-) OOH-} phosphatidylethanolamine (PE) was identified from various phospholipids comprising the majority of lipid peroxides as the most important signal of ferroptosis [27]. Specifically, the process of AA to AA-CoA is driven by acyl-CoA synthetase long-chain family 4 (ACSL4) [28, 29], after which AA-CoA is converted to AA-PE by lysophosphatidylcholine acyltransferase 3- (LPCAT3-) promoted esterification [30]. Then, the final step to forming AA-OOH-PE requires the oxidation of AA-PE catalyzed by lipoxygenases (LOXs) [31]. The esterification and oxidation steps can also occur in a reversed order. Eventually, the uncontrolled accumulation of AA-OOH-PE induces ferroptosis. Some studies suggest that the expression of ACSL4 may, to some extent, reflect the sensitivity of a cell to ferroptosis and can be used as a marker [28].

The production of lipid peroxides is iron-dependent. Iron is involved in lipid oxidation in the following three possible ways: (I) the Fenton reaction which is an inorganic, nonenzymatic catalyzed reaction, where ferrous iron donates electrons to $\mathrm{O}_{2}$ or $\mathrm{H}_{2} \mathrm{O}_{2}$ to promote the production of reactive oxygen species (ROS) and lipid peroxides; (II) lipid autoxidation by an iron-catalyzed enzymatic reaction; and (III) AA oxidation by an iron-containing LOX-catalyzed reaction [8]. In addition, there is evidence that the cell sensitivity of ferroptosis is affected by alterations in genes regulating iron homeostasis (e.g., IREB2, FBXL5, and FTH1 influence the cell sensitivity of erastin-induced ferroptosis) and in the extracellular concentration of iron [8]. For example, in rodent models, high-iron diets increase their sensitivity to ferroptosis through increasing the extracellular concentration of iron $[32,33]$. Some proteins such as heat shock protein family B member 1 (HSPB1), which can decrease the intracellular level of iron, influence the ferroptotic sensitivity as well [34]. The nuclear receptor coactivator 4(NCOA4-) mediated ferritinophagy plays a vital role in releasing iron from ferritin. During ferritinophagy, NCOA4 binds to ferritin and delivers it to lysosomes for degradation to release iron [35]. Then, the high concentration of intracellular iron may further induce ferroptosis.

2.2. Lipid Peroxide Scavenging and Ferroptosis. GPX4 is the major scavenger of lipid peroxides in cells, and it is a member of the selenoprotein family. Selenium is indispensable for the oxidoreductase function of GPX4 because selenium contributes to the nucleophilicity of selenoproteins $[36,37]$. Thus, a deficiency of selenium in serum or cytoplasm is likely to impair the function of GPX4, eventually causing the accumulation of lipid peroxides and then ferroptosis [38]. GPX4 has eight nucleophilic amino acids, one of which is the selenocysteine (Sec) at its active site. RAS-selective lethal 3 (RSL3) contains a chloroacetamide moiety that can react with the nucleophilic amino acid residues on GPX4, and the binding of RSL3 to GPX4 leads to inactivation of GPX4, making RSL3 a ferroptosis inducer [39].

Glutathione (GSH) is a cofactor of GPX4 and consists of three subunits: glutamate, glycine, and cysteine (a reduced form of cystine). Erastin suppresses the activity of GPX4 by inhibiting the $x_{c}{ }^{-}$system, which imports cystine into the cell [3]. Inadequate supply of cystine due to an inhibition of the $x_{c}{ }^{-}$system leads to decreased production of cysteine and the depletion of GSH, which will eventually suppress the normal activity of GPX4 in preventing ferroptosis [27].

Ferroptosis inducing 56 (FIN56) facilitates the degradation of GPX4, and 1,2-dioxolane (FINO2) inactivates GPX4 [8]. The underlying mechanisms of FIN56 and FINO2 in the degradation and inactivation of GPX4 are unknown. Additionally, FINO2 can oxidize ferrous iron to produce ROS and oxidize lipids to produce lipid peroxides, which together induce ferroptosis $[40,41]$.

The mevalonate pathway is of vital importance in ferroptosis. It is the most relevant cellular metabolic pathway that affects the biosynthesis of selenoproteins and other antioxidant molecules such as ubiquinol [42]. The metabolic intermediate of the mevalonate pathway, isopentenyl pyrophosphate, is indispensable for the biosynthesis of several molecules including ubiquinol [43]. Ubiquinol inhibits lipid peroxidation in the plasma membrane and blocks ferroptosis. In addition to its GPX4-degrading ability, FIN56 
disturbs ubiquinone synthesis through the mevalonate pathway [44]. An antagonist of FIN56 is the ferroptosis suppressor protein 1 (FSP1, previously called AIFM2), which is one of the enzymes catalyzing the transformation of ubiquinone to ubiquinol [45]. Notably, although ubiquinone exists in almost all lipid membranes, the FSP1-dependent modification of ubiquinone can only protect against lipid peroxidation in locations exclusive of mitochondria [45].

\subsection{Ferroptosis and Other Forms of Cell Death}

2.3.1. Common Features. Although it is widely acknowledged that ferroptosis is genetically, biochemically, and morphologically distinct from other RCD processes such as apoptosis, necroptosis, and parthanatos, it shares a few common features with them. As has been mentioned above, NCOA4 contributes to ferroptosis via ferritinophagy to release more iron from ferritin [35]. Additionally, there is evidence that ferroptosis is accelerated when NCOA4 expression is forced to increase by cDNA transfection [46], but limited when NCOA4 is genetically depleted [46]. However, ferritinophagy is mechanistically a selective autophagy process [47]. Thus, some studies support the idea that the activation of ferroptosis depends on the induction of autophagy [46-49]. Intrinsically disordered proteins (IDPs) and IDP regions (IDPRs) are characterized by a lack of fixed 3D structure, which allows for promiscuous interaction and regulation with other proteins via structurally unrelated messengers [50]. IDPs and IDPRs are common in apoptosis, autophagy, and necroptosis [51]. Studies have found that approximately $40 \%$ of the proteins involved in ferroptosis have some disordered regions with $<30$ residues and $\sim 15 \%$ presented long-range disordered regions of $>90$ residues similar to IDPs in the prevalence and distribution of long disordered regions. This implied that IDPs and IDPRs are common features among ferroptosis and other RCDs [3, 51, 52]. Additionally, this result was in favor of the hypothesis that signaling proteins without a specific structure are likely to be involved in the onset of ferroptosis [52]. When SLC7A11, the key component of the $x_{c}{ }^{-}$system, is inhibited by erastin, the depletion of intracellular GSH [3] and the inactivation of GPX4 lead to ferroptosis [27]. However, GSH and GPX4 are both modulators in apoptosis, necroptosis, and autophagy [53, 54]. Additionally, other ferroptosis regulators such as NRF2 [55], P53 [56], and ACSL4 $[28,57,58]$ may also be potential regulators of apoptosis, necroptosis, and autophagy.

\subsubsection{Communications through Mitochondrial Dysfunction} and Endoplasmic Reticulum Stress. Mitochondrial impairment, endoplasmic reticulum (ER) stress, and inhibition of the $x_{c}{ }^{-}$system have been observed in ferroptosis [59-61].

Decreases in mitochondrial membrane potential (MMP) and increases in mitochondrial transition pore permeability initiate the processes of apoptosis and necrosis [62]. However, ferroptosis can also happen due to the mitochondrial dysfunction causing disturbance in mitochondrial iron homeostasis. A research shows that decreased MMP, accelerated ferritinophagy, and more ROS production in mitochondria caused by dihydroartemisinin (DHA) induce ferroptosis in acute myeloid leukemia cells [20]. However, iron-sulfur cluster assembly enzyme (ISCU), a mitochondrial protein, can alleviate the toxicity of DHA by regulating iron metabolism, preserving mitochondrial function, and increasing the level of GSH [20]. Thus, it is possible that when mitochondria are damaged, ferroptosis occurs due to the dysregulation of mitochondrial iron homeostasis along with other RCD processes.

Oxytosis, also named oxidative glutamate toxicity, is a glutamate-induced cell death mediated by a block of the $x_{c}{ }^{-}$ system [63]. Notably, dysfunctional $x_{c}^{-}$system causing GSH depletion also happens in ferroptosis $[8,20,64]$. Research demonstrated that ferroptosis shares part of its pathway with oxytosis where BID (a proapoptotic protein) mediates cell death from mitochondrial dysfunction [10, 65]. Specifically, in both cases of BID knockout and using BI-6c9 (a BID inhibitor), the erastin-induced (ferroptosis) and the glutamate-induced (oxytosis) cell deaths were blocked, whereas overexpressed BID promoted cell death [65]. The ferroptosis inhibitor ferrostatin-1 (Fer-1) partially prevents oxytosis through blocking BID translocation to mitochondria but it is unable to rescue damages caused by BID that are already in mitochondria. However, BI- $6 \mathrm{c} 9$ can counteract BID before and after its translocation to mitochondria. The BID pathway in ferroptosis and oxytosis is also detected in mouse embryonic fibroblasts [65].

Although there is not enough evidence to suggest that ferroptosis further induces apoptosis in cells, there are experiments demonstrating that ferroptotic agents not only induce ferroptosis but also enhance tumor necrosis factor-related apoptosis-inducing ligand- (TRAIL-) induced apoptosis [56]. TRAIL is capable of inducing apoptosis in malignant human cells but not in the majority of normal cells, which is a pharmacologically preferred property [66]. When treating human cancer cells with artesunate (ART, a ferroptosis inducer) or erastin together with TRAIL, the cytotoxicity of TRAIL is enhanced through an increase in ER stressinduced p53-independent PUMA (p53 upregulated modulator of apoptosis) expression [56]. However, TRAIL has no effects on ferroptotic agents-induced lipid peroxidation when they are supplied to cancer cells simultaneously. Additionally, ferroptosis inhibitors Fer-1 and liproxstatin- 1 cannot prevent ER stress or the synergistic cytotoxicity of ER stress and TRAIL [56]. Interestingly, iron chelator deferoxamine (DFO) is the only ferroptotic inhibitor that can suppress ART-induced (but not erastin-induced) ER stress [56]. The varied results of different ferroptosis inhibitors indicate two things: one is that although ferroptosis and apoptosis may share common pathways leading to cell death, they are independent; the other is that the different ferroptosis inhibitors may act in different ways. Thus, drug combination of inhibitors of ferroptosis and apoptosis is suggested in preventing cell death to achieve better protective effect.

\section{Ferroptosis in Diabetes Mellitus}

It was reported that $9.3 \%$ of Americans (approximately 29.1 million persons) were diagnosed with diabetes in 2014 [67], and it was estimated that 86.1 million adults in the United 
States have prediabetes [67]. Islet $\beta$-cell failure and peripheral insulin resistance are the main pathological manifestations of diabetes mellitus. To be specific, type 1 diabetes mellitus is attributed to an absolute insulin deficiency due to $\beta$-cell destruction, while type 2 diabetes mellitus (T2DM) is attributed to a progressive insulin secretory defect on the background of insulin resistance [68]. The complications of T2DM are implicated in almost every tissue of the body especially the cardiovascular system, the optical system, and the renal system [69]. The pathogenesis of T2DM is still largely unknown, and so is its association with ferroptosis. To date, only a small number of studies have explored the relationship between ferroptosis and T2DM. In this section, we will review the potential role of ferroptosis in T2DM.

Pancreatic islet $\beta$-cells are susceptible to ferroptosis. Some studies indicate that pancreatic $\beta$-cells express a low level of antioxidant enzymes such as superoxide dismutase (SOD), GSH peroxidase, and catalase [70]. Hence, they are susceptible to oxidative stress because ROS is prone to accumulate. ROS accumulation can trigger many forms of deterioration, including ferroptosis. A study showed significantly reduced glucose-stimulated insulin secretion (GSIS) capacity in human islet $\beta$-cells when they were treated with the ferroptosis inducer erastin (but not RSL3) in vitro [71]. Conversely, pretreatment with a ferroptosis inhibitor, Fer-1 or DFO, rescued the damage to GSIS [71].

ROS concentration in $\beta$-cells can be raised by arsenic. There is evidence that chronic exposure to arsenic is a significant risk factor for developing T2DM [72, 73]. An experiment using MIN6 cells, mice pancreatic islet $\beta$-cell line, showed that ferroptosis was involved in pancreatic islet $\beta$ cells injury caused by arsenic via increased intracellular iron concentration and accumulated lipid peroxides resulting in RCD. Specifically, mitochondrial damage caused by $\mathrm{NaAsO} 2$ produced excessive mitochondrial ROS (MtROS), which further led to MtROS-dependent autophagy and increased intracellular concentration of iron. This eventually resulted in ferroptosis in MIN6 cells and impaired insulin secretion [74]. In addition, blocking this MtROS-mediated pathway promoted the insulin secretion of islet $\beta$-cells [74]. Another study showed that curcumin and (-)-epigallocatechin-3-gallate (EGCG), two polyphenols, can protect murine MIN6 pancreatic $\beta$-cells from iron toxicity and erastin-induced ferroptosis by acting as iron chelators and preventing GSH depletion and lipid peroxidation [75]. In addition to their iron-chelating properties, some polyphenols may also function as an antioxidant [76].

Taken together, ferroptosis is associated with insulin secretion dysfunction in pancreatic $\beta$-cells. The function of pancreatic islets can be impaired by proferroptotic factors even before $\beta$-cells die. Thus, monitoring and control of ferroptosis-related factors may facilitate early diagnosis and therapy of T2DM.

3.1. Induction of Iron Accumulation in T2DM. Ferroptosis is directly associated with the body level of ferritin. Epidemiological studies have revealed the potential association between excessive body iron storage and T2DM [75, 77, 78]. The development of insulin resistance in the association between iron and T2DM has been revealed in early studies $[78,79]$. The current hypothesis is that the higher the body iron storage, the higher the risk in developing T2DM [80, 81]. For example, a mouse model of hereditary hemochromatosis which resulted in iron overloaded revealed iron deposition, increased fatty acid oxidation, and decreased glucose oxidation in the skeletal muscle, which eventually aggravates insulin resistance $[78,79]$. So hemochromatosis is possibly related to diabetes. Nonetheless, a longitudinal study on overweight/obese individuals with an impaired glucose tolerance test showed no association between body iron storage and the incidence of diabetes $[82,83]$. A possible explanation is that in this case, obesity is the dominant factor in diabetes development.

From the perspective of ameliorating diabetes, there are studies demonstrating that improved insulin secretion and insulin sensitivity as well as better control of blood glucose were observed after reducing the level of body iron storage $[84,85]$.

However, it cannot be ignored that calculating the body iron storage from the level of serum ferritin is not fully reliable, because ferritin also rises in inflammation, cancer, and liver disease [86]. Thus, it is still unclear whether high ferritin in the blood is a cause or result of diabetes [78]. Nevertheless, serum ferritin level test can still be used in the early diagnosis of T2DM and gestational diabetes [87-90].

Iron-sulfur (Fe-S) clusters are contained in the enzymes involved in the modification of tRNA at position 37 [91]. Since most tRNAs need to be modified at position 37, which is adjacent to the $3^{\prime}$ position of the anticodon, Fe-S clusters are indispensable for tRNA modification and protein translation. For example, Fe-S clusters are involved in forming 2-methylthio-N6-threonyl carbamooyladenosine $\left(\mathrm{ms}^{2} \mathrm{t}^{6} \mathrm{~A}\right)$ at position 37 in tRNA ${ }^{\text {Lys }}(\mathrm{UUU})[59,92]$. When cells lack $\mathrm{ms}^{2} \mathrm{t}^{6} \mathrm{~A} 37$ due to Fe-S cluster deficiency, proteins including proinsulin are mistranslated, which may further trigger ER stress $[59,93]$. The abnormal insulin synthesis and secretion as well as ER stress due to the mistranslated proinsulin contributes to the development of T2DM [59]. Meanwhile, Fe$S$ clusters regulate mitochondrial iron homeostasis in mitochondria [91]. The mitochondrial iron accumulation caused by Fe-S cluster deficiency can further lead to iron-mediated ROS generation inside mitochondria [94] and then ferroptosis cell death due to lipid peroxides accumulation [20]. This induction of ferroptosis causes RCD in $\beta$-cells accelerating the development of T2DM [74]. Also, ROS directly impairs insulin synthesis and secretion in the development of T2DM [95] (Figure 1).

3.2. Double-Faceted Effects of Selenoproteins and ACSL4 on T2DM Promotion. Selenoproteins and ACSL4 are groups of enzymes involved in the regulation of lipid peroxides. These enzymes are not limited in ferroptosis. Actually, they are active in many physiological reactions and potentially have an effect on the pathogenesis of T2DM.

Although ROS is often considered a contributor to the pathogenesis of T2DM, ROS is intrinsically a signaling molecule that is indispensable in the downstream insulininduced signaling pathway to assist glucose disposition. The 


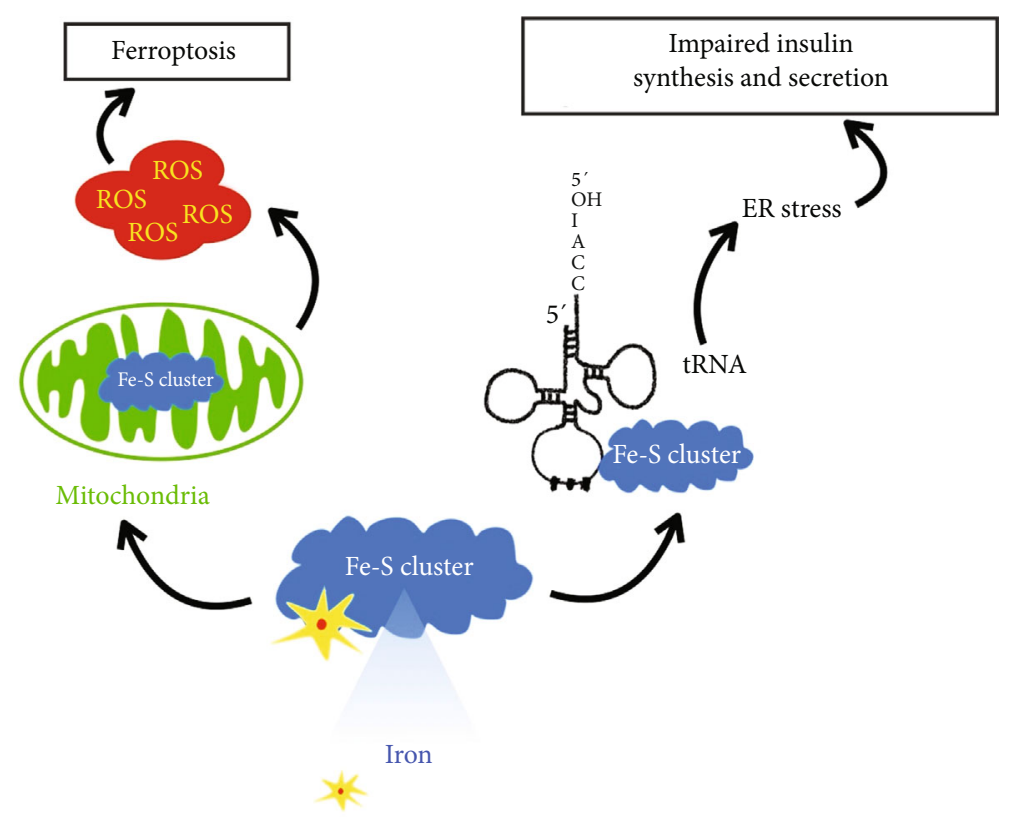

FIgURE 1: The pathway of Fe-S cluster deficiency in T2DM. Fe-S cluster deficiency affects the modification and function of tRNA, resulting in mistranslated proinsulin. This mistranslation can lead to ER stress and impaired insulin synthesis and secretion. Also, iron accumulation and more ROS production due to Fe-S cluster deficiency in mitochondria promote lipid peroxidation, further inducing ferroptosis in $\beta$-cells. Additionally, ROS directly impairs insulin synthesis and secretion.

$\mathrm{Gpx}^{-/-}$mice were generated through inserting a $1.1 \mathrm{~kb}$ fragment of neomycin resistance gene cassette into the unique SacII site within the first exon of Gpx1. This $\mathrm{Gpx}^{-/-}$mice model plays a unique role in elucidating the contribution of Gpx1 in the protection against situations of oxidative stress $[96,97]$. An experiment on $\mathrm{Gpx}^{-/-}$mice demonstrated the positive effect of ROS in enhancing insulin sensitivity [98]. GPX1 and GPX4 are both from the same selenoprotein family and are both antioxidant enzymes. This explains why GPX1 knockout resulted in elevated ROS production, but how does ROS promote insulin sensitivity? Further research showed that ROS activated the PI3K/Akt signaling pathway, which is key in the downstream of the insulininduced pathway leading to glucose disposition $[98,99]$. This was especially observed in the skeletal muscle. Thus, GPX1 knockout increased insulin sensitivity through ROSmediated enhancement of the PI3K/Akt signaling pathway, leading to more glucose uptake in muscle [98]. Although GPX1 and GPX4 are from the same protein family, the deficiency of GPX 4 in islet $\beta$-cells may trigger T2DM due to ferroptosis while the deficiency of GPX1 enhances peripheral insulin sensitivity.

As has been mentioned above, ACSL4 catalyzes AA into AA-CoA to produce lipid peroxides in ferroptosis. Upregulated expression of ACSL4 was observed in mice fed with a high-fat diet [100]. In that same study, adipocyte-specific ablation of ACSL4 (Ad-KO) in mice fed with a high-fat diet was found to protect the mice from developing insulin resistance [100]. However, another experiment demonstrated that ACSL4 proteins are present in $\beta$-cells in human and rat pancreatic islets and are more concentrated around insulin secretory granules and mitochondria than at other intra- cellular organelles [101]. This result suggests that ACSL4 participates in insulin secretion by modifying fatty acids in insulin secretory granules and mitochondria [101]. Thus, more experiments on the function of ACSL4 are needed to delineate the role of ACSL4 in T2DM pathogenesis and the potential of targeting ACSL4 in the treatment of T2DM (Figure 2).

3.3. Ferroptosis and Myocardial Diseases in T2DM. Under diabetic conditions, dysregulation blood sugar levels may cause complications in almost every tissue of the body especially the cardiovascular system, the optical system, and the renal system [69]. In fact, evidence indicates that ferroptosis is involved in IRI $[15,102,103]$. Hence, organs that are more likely to suffer from ischemia (e.g., the cardiomyocytes) in T2DM are more likely to undergo ferroptosis [102, 104].

The incidence of myocardial ischemia in diabetes is 2.45 to 2.99 times higher than that in nondiabetes [105]. The prevalence of silent myocardial ischemia is $20 \sim 30 \%$ in asymptomatic patients with T2DM [106]. Thus, T2DM can be a risk factor for myocardial IRI. It has been confirmed that iron overloaded happens in cardiomyocytes and nonmyocytes during IRI in vivo [107]. This study also confirmed that ferroptosis can be induced in primary cultures of adult mouse cardiomyocytes using either $\mathrm{Fe}^{3+}$ or either of two ferroptosis inducers: erastin or RSL3 [107]. A recent study has demonstrated that inhibition of ferroptosis by Fer-1 during myocardial ischemia and reperfusion in diabetic rats can alleviate ER stress and myocardial damage with further in-depth experiments indicating that ferroptosis is involved in myocardial IRI through ER stress [102]. These findings suggested that during the process of myocardial IRI, ferroptosis and ER 


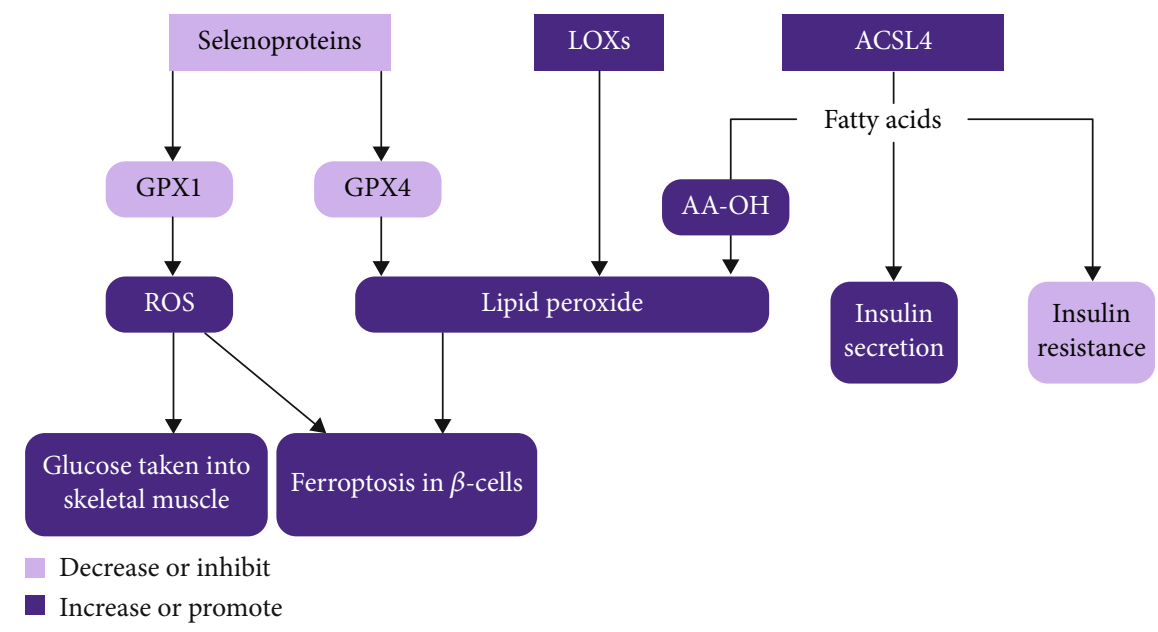

FIgURE 2: Selenoproteins, LOXs, and ACSL4 affect pathogenesis of DM in different ways. GPX1 and GPX4 are both selenoproteins. A decrease in GPX1 or GPX4 causes accumulation of ROS and lipid peroxides in $\beta$-cells. Excessive ROS and lipid peroxides in turn induce ferroptosis in $\beta$-cells. ROS also promotes glucose intake into skeletal muscles. An increase in LOXs or ACSL4 produces excessive lipid peroxides and then ferroptosis in $\beta$-cells as well. Although ACSL4 can promote insulin secretion in $\beta$-cells, it aggravates peripheral insulin resistance.

stress enhanced each other in causing myocardial injury [102]. Another study also demonstrated that Fer-1-treated mice showed reduced infarct size and serum markers of myocardial injury in IRI in vivo [15]. Some other ferroptosis inhibitors also have the ability to alleviate IRI. For example, iron chelator DFO reduced infarct size [15, 25], and the overexpression of GPX4 in mitochondria preserved mitochondrial and contractile function after global ischemia/reperfusion $(\mathrm{I} / \mathrm{R})$ in isolated, perfused mouse hearts [108]. However, these studies only show that ferroptosis is involved in IRI, and the specific role of ferroptosis needs more exploration.

Diabetic cardiomyopathy (DCM) is not an uncommon disease among diabetes [109]. Oxidative stress and impaired antioxidant system under the hyperglycemic condition are the basis of the pathogenesis of DCM $[55,110,111]$. Since an imbalance of the antioxidant system usually leads to excessive ROS production, consequent ferroptosis, apoptosis, inflammation, and fibrosis may occur in myocardial cells [55]. Nuclear factor-erythroid 2 p45-related factor 2 (NRF2) plays a pivotal role in maintaining cellular redox by its regulation of multiple antioxidants [112]. The regulations include almost all the genes encoding antioxidants implicated in ferroptosis such as the genes for glutathione regulation, NADPH regeneration, lipid peroxidation, and iron regulation $[113,114]$. Loss of selenoprotein expression is in part counteracted by the NRF2-dependent system [38]. Recent studies indicate that activating NRF2 to suppress ferroptosis can be a potential therapeutic target for DCM in animal models $[115,116]$. Rutin is both an NRF2 activator and a phytochemical with multiple pharmacological activities including antidiabetic, antioxidative, and free radicalscavenging bioactivities. It has been shown to be effective in alleviating DCM in animal models of both type 1 and type 2 diabetes [117-119]. However, how NRF2 activation alters ferroptosis in the pathogenesis and development of DCM remains unclear [119].

\section{Conclusion}

Ferroptosis features intracellular iron overloaded and lipid peroxides. Iron and enzymes such as LOXs, ACSL4, and LPCAT3 together produce excessive lipid peroxides (especially AA-OOH-PE) in cells, causing ferroptosis. Thus, the levels of iron and those lipid oxidases may implicate the cellular sensitivity to ferroptosis. GPX4 functions as a lipid reductase. Molecules inhibiting the activation of GPX4, such as RSL3 via combining with its active site, erastin via depleting its cofactor GSH, and FIN56 via facilitating its degradation, also induce ferroptosis. Moreover, IDPs/IDPRs, mitochondrial dysfunction, and ER stress are what correlate ferroptosis with other RCDs.

Ferroptosis is widely investigated in its role in both physiologic and pathogenic processes but its role in T2DM has not been extensively studied. Since $\beta$-cells lack a strong antioxidation mechanism, they are possibly susceptible to ferroptosis. Evidence has shown the involvement of ferroptosis in GSIS impairment and arsenic-induced pancreatic islet cell damage. High concentrations of serum iron can be a risk factor of developing T2DM. Hence, monitoring and control of factors related to ferroptosis might be promising measures in the early diagnosis and therapy of T2DM. However, additional studies on ferroptosis and its involvement in T2DM are needed to identify the appropriate diagnostic and therapeutic targets.

\section{Data Availability}

All data used and/or analysed during the present study are available from the corresponding author on reasonable request.

\section{Conflicts of Interest}

The authors declare that they have no conflicts of interest. 


\section{Acknowledgments}

This work was supported by Ningbo Science and Technology Innovation Team Program (2014B82002 to SB), Ningbo 3315 Program (2013A-10-G to SB), the National Natural Science Foundation of China (81370165 to SB), Ningbo Science and Technology Huiming Program (201701CX-D02072 to YC), Ningbo Natural Science Foundation (2015A610217 to YC), Fang Runhua Fund of Hong Kong, and K. C. Wong Magna Fund in Ningbo University.

\section{References}

[1] Y. Fuchs and H. Steller, "Programmed cell death in animal development and disease," Cell, vol. 147, no. 4, pp. 742-758, 2011.

[2] C. B. Thompson, "Apoptosis in the pathogenesis and treatment of disease," Science, vol. 267, no. 5203, pp. 1456-1462, 1995.

[3] S. J. Dixon, K. M. Lemberg, M. R. Lamprecht et al., "Ferroptosis: an iron-dependent form of nonapoptotic cell death," Cell, vol. 149, no. 5, pp. 1060-1072, 2012.

[4] A. Pena-Blanco and A. J. Garcia-Saez, "Bax, Bak and beyond mitochondrial performance in apoptosis," The FEBS Journal, vol. 285, no. 3, pp. 416-431, 2018.

[5] T. Bergsbaken, S. L. Fink, and B. T. Cookson, "Pyroptosis: host cell death and inflammation," Nature Reviews. Microbiology, vol. 7, no. 2, pp. 99-109, 2009.

[6] D. E. Christofferson and J. Yuan, "Necroptosis as an alternative form of programmed cell death," Current Opinion in Cell Biology, vol. 22, no. 2, pp. 263-268, 2010.

[7] V. K. Vyas, C. Chintha, and M. R. Pandya, "Biology and medicinal chemistry approaches towards various apoptosis inducers," Anti-Cancer Agents in Medicinal Chemistry, vol. 13, no. 3, pp. 433-455, 2013.

[8] P. Lei, T. Bai, and Y. Sun, "Mechanisms of ferroptosis and relations with regulated cell death: a review," Frontiers in Physiology, vol. 10, p. 139, 2019.

[9] M. Matsushita, S. Freigang, C. Schneider, M. Conrad, G. W. Bornkamm, and M. Kopf, "T cell lipid peroxidation induces ferroptosis and prevents immunity to infection," The Journal of Experimental Medicine, vol. 212, no. 4, pp. 555-568, 2015.

[10] C. T. Salisbury-Ruf, C. C. Bertram, A. Vergeade et al., "Bid maintains mitochondrial cristae structure and function and protects against cardiac disease in an integrative genomics study," eLife, vol. 7, 2018.

[11] B. R. Stockwell, J. P. Angeli, H. Bayir et al., "Ferroptosis: a regulated cell death nexus linking metabolism, redox biology, and disease," Cell, vol. 171, no. 2, pp. 273-285, 2017.

[12] S. J. Guiney, P. A. Adlard, A. I. Bush, D. I. Finkelstein, and S. Ayton, "Ferroptosis and cell death mechanisms in Parkinson's disease," Neurochemistry International, vol. 104, pp. 3448, 2017.

[13] G. Priante, L. Gianesello, M. Ceol, D. Del Prete, and F. Anglani, "Cell death in the kidney," International Journal of Molecular Sciences, vol. 20, no. 14, 2019.

[14] T. Müller, C. Dewitz, J. Schmitz et al., "Necroptosis and ferroptosis are alternative cell death pathways that operate in acute kidney failure," Cellular and Molecular Life Sciences, vol. 74, no. 19, pp. 3631-3645, 2017.
[15] X. Fang, H. Wang, D. Han et al., "Ferroptosis as a target for protection against cardiomyopathy," Proceedings of the National Academy of Sciences of the United States of America, vol. 116, no. 7, pp. 2672-2680, 2019.

[16] D. Martin-Sanchez, O. Ruiz-Andres, J. Poveda et al., "Ferroptosis, but not necroptosis, is important in nephrotoxic folic acid-induced AKI," Journal of the American Society of Nephrology, vol. 28, no. 1, pp. 218-229, 2017.

[17] A. Linkermann, R. Skouta, N. Himmerkus et al., "Synchronized renal tubular cell death involves ferroptosis," Proceedings of the National Academy of Sciences of the United States of America, vol. 111, no. 47, pp. 16836-16841, 2014.

[18] H. Kasai, "Analysis of a form of oxidative DNA damage, 8hydroxy-2'-deoxyguanosine, as a marker of cellular oxidative stress during carcinogenesis," Mutation Research, vol. 387, no. 3, pp. 147-163, 1997.

[19] S. Toyokuni, "Iron and carcinogenesis: from Fenton reaction to target genes," Redox Report, vol. 7, no. 4, pp. 189-197, 2002.

[20] J. Du, T. Wang, Y. Li et al., "DHA inhibits proliferation and induces ferroptosis of leukemia cells through autophagy dependent degradation of ferritin," Free Radical Biology \& Medicine, vol. 131, pp. 356-369, 2019.

[21] R. Kang, G. Kroemer, and D. Tang, "The tumor suppressor protein p53 and the ferroptosis network," Free Radical Biology \& Medicine, vol. 133, pp. 162-168, 2019.

[22] L. Jiang, N. Kon, T. Li et al., "Ferroptosis as a p53-mediated activity during tumour suppression," Nature, vol. 520, no. 7545, pp. 57-62, 2015.

[23] Y. Ou, S. J. Wang, D. Li, B. Chu, and W. Gu, "Activation of SAT1 engages polyamine metabolism with p53-mediated ferroptotic responses," Proceedings of the National Academy of Sciences of the United States of America, vol. 113, no. 44, pp. E6806-E6812, 2016.

[24] W. Hu, C. Zhang, R. Wu, Y. Sun, A. Levine, and Z. Feng, "Glutaminase 2, a novel p53 target gene regulating energy metabolism and antioxidant function," Proceedings of the National Academy of Sciences of the United States of America, vol. 107, no. 16, pp. 7455-7460, 2010.

[25] M. Gao, P. Monian, N. Quadri, R. Ramasamy, and X. Jiang, "Glutaminolysis and transferrin regulate ferroptosis," Molecular Cell, vol. 59, no. 2, pp. 298-308, 2015.

[26] Y. Xie, S. Zhu, X. Song et al., "The tumor suppressor p53 limits ferroptosis by blocking DPP4 activity," Cell Reports, vol. 20, no. 7, pp. 1692-1704, 2017.

[27] V. E. Kagan, G. Mao, F. Qu et al., "Oxidized arachidonic and adrenic PEs navigate cells to ferroptosis," Nature Chemical Biology, vol. 13, no. 1, pp. 81-90, 2017.

[28] H. Yuan, X. Li, X. Zhang, R. Kang, and D. Tang, "Identification of ACSL4 as a biomarker and contributor of ferroptosis," Biochemical and Biophysical Research Communications, vol. 478, no. 3, pp. 1338-1343, 2016.

[29] S. Doll, B. Proneth, Y. Y. Tyurina et al., "ACSL4 dictates ferroptosis sensitivity by shaping cellular lipid composition," Nature Chemical Biology, vol. 13, no. 1, pp. 91-98, 2017.

[30] S. J. Dixon, G. E. Winter, L. S. Musavi et al., "Human haploid cell genetics reveals roles for lipid metabolism genes in nonapoptotic cell death," ACS Chemical Biology, vol. 10, no. 7, pp. 1604-1609, 2015.

[31] R. Shintoku, Y. Takigawa, K. Yamada et al., "Lipoxygenasemediated generation of lipid peroxides enhances ferroptosis 
induced by erastin and RSL3," Cancer Science, vol. 108, no. 11, pp. 2187-2194, 2017.

[32] H. Wang, P. An, E. Xie et al., "Characterization of ferroptosis in murine models of hemochromatosis," Hepatology, vol. 66, no. 2, pp. 449-465, 2017.

[33] G. S. Panjrath, V. Patel, C. I. Valdiviezo, N. Narula, J. Narula, and D. Jain, "Potentiation of doxorubicin cardiotoxicity by iron loading in a rodent model," Journal of the American College of Cardiology, vol. 49, no. 25, pp. 2457-2464, 2007.

[34] X. Sun, Z. Ou, M. Xie et al., "HSPB1 as a novel regulator of ferroptotic cancer cell death," Oncogene, vol. 34, no. 45, pp. 5617-5625, 2015.

[35] J. D. Mancias, X. Wang, S. P. Gygi, J. W. Harper, and A. C. Kimmelman, "Quantitative proteomics identifies NCOA4 as the cargo receptor mediating ferritinophagy," Nature, vol. 509, no. 7498, pp. 105-109, 2014.

[36] J. T. Rotruck, A. L. Pope, H. E. Ganther, A. B. Swanson, D. G. Hafeman, and W. Hoekstra, "Selenium: biochemical role as a component of glutathione peroxidase," Science, vol. 179, no. 4073 , pp. $588-590,1973$.

[37] L. Flohe, W. A. Gunzler, and H. H. Schock, "Glutathione peroxidase: a selenoenzyme," FEBS Letters, vol. 32, no. 1, pp. 132-134, 1973.

[38] J. P. Friedmann Angeli, "Selenium and GPX4, a vital symbiosis," Free Radical Biology \& Medicine, vol. 127, pp. 153-159, 2018.

[39] W. S. Yang, K. J. Kim, M. M. Gaschler, M. Patel, M. S. Shchepinov, and B. R. Stockwell, "Peroxidation of polyunsaturated fatty acids by lipoxygenases drives ferroptosis," Proceedings of the National Academy of Sciences of the United States of America, vol. 113, no. 34, pp. E4966-E4975, 2016.

[40] R. P. Abrams, W. L. Carroll, and K. A. Woerpel, "Five-membered ring peroxide selectively initiates ferroptosis in cancer cells," ACS Chemical Biology, vol. 11, no. 5, pp. 1305-1312, 2016.

[41] M. M. Gaschler, A. A. Andia, H. Liu et al., "FINO2 initiates ferroptosis through GPX4 inactivation and iron oxidation," Nature Chemical Biology, vol. 14, no. 5, pp. 507-515, 2018.

[42] B. Moosmann and C. Behl, "Selenoproteins, cholesterollowering drugs, and the consequences: revisiting of the mevalonate pathway," Trends in Cardiovascular Medicine, vol. 14, no. 7, pp. 273-281, 2004.

[43] S. A. Holstein and R. J. Hohl, "Isoprenoids: remarkable diversity of form and function," Lipids, vol. 39, no. 4, pp. 293-309, 2004.

[44] K. Shimada, R. Skouta, A. Kaplan et al., "Global survey of cell death mechanisms reveals metabolic regulation of ferroptosis," Nature Chemical Biology, vol. 12, no. 7, pp. 497-503, 2016.

[45] B. R. Stockwell, "A powerful cell-protection system prevents cell death by ferroptosis," Nature, vol. 575, no. 7784, pp. 597-598, 2019.

[46] W. Hou, Y. Xie, X. Song et al., "Autophagy promotes ferroptosis by degradation of ferritin," Autophagy, vol. 12, no. 8, pp. 1425-1428, 2016.

[47] R. Kang and D. Tang, "Autophagy and ferroptosis - what's the connection?," Current pathobiology reports, vol. 5, no. 2, pp. 153-159, 2017.

[48] M. Gao, P. Monian, Q. Pan, W. Zhang, J. Xiang, and X. Jiang, "Ferroptosis is an autophagic cell death process," Cell Research, vol. 26, no. 9, pp. 1021-1032, 2016.
[49] S. Torii, R. Shintoku, C. Kubota et al., "An essential role for functional lysosomes in ferroptosis of cancer cells," The Biochemical Journal, vol. 473, no. 6, pp. 769-777, 2016.

[50] B. Xue, A. K. Dunker, and V. N. Uversky, "Orderly order in protein intrinsic disorder distribution: disorder in 3500 proteomes from viruses and the three domains of life," Journal of Biomolecular Structure \& Dynamics, vol. 30, no. 2, pp. 137-149, 2012.

[51] Z. Peng, B. Xue, L. Kurgan, and V. N. Uversky, "Resilience of death: intrinsic disorder in proteins involved in the programmed cell death," Cell Death and Differentiation, vol. 20, no. 9, pp. 1257-1267, 2013.

[52] M. B. Djulbegovic and V. N. Uversky, "Ferroptosis - an ironand disorder-dependent programmed cell death," International Journal of Biological Macromolecules, vol. 135, pp. 1052-1069, 2019.

[53] M. Maiorino, M. Conrad, and F. Ursini, "GPx4, lipid peroxidation, and cell death: discoveries, rediscoveries, and open issues," Antioxidants \& Redox Signaling, vol. 29, no. 1, pp. 61-74, 2018.

[54] Y. Sun, Y. Zheng, C. Wang, and Y. Liu, "Glutathione depletion induces ferroptosis, autophagy, and premature cell senescence in retinal pigment epithelial cells," Cell Death of Disease, vol. 9, no. 7, p. 753, 2018.

[55] M. S. Shah and M. Brownlee, "Molecular and cellular mechanisms of cardiovascular disorders in diabetes," Circulation Research, vol. 118, no. 11, pp. 1808-1829, 2016.

[56] S. H. Hong, D. H. Lee, Y. S. Lee et al., "Molecular crosstalk between ferroptosis and apoptosis: emerging role of ER stress-induced p53-independent PUMA expression," Oncotarget, vol. 8, no. 70, pp. 115164-115178, 2017.

[57] U. D. Orlando, A. F. Castillo, M. A. Dattilo, A. R. Solano, P. M. Maloberti, and E. J. Podesta, "Acyl-CoA synthetase-4, a new regulator of $\mathrm{mTOR}$ and a potential therapeutic target for enhanced estrogen receptor function in receptorpositive and -negative breast cancer," Oncotarget, vol. 6, no. 40, pp. 42632-42650, 2015.

[58] C. H. Jung, S. H. Ro, J. Cao, N. M. Otto, and D. H. Kim, "mTOR regulation of autophagy," FEBS Letters, vol. 584, no. 7, pp. 1287-1295, 2010.

[59] A. G. Torres, E. Batlle, and L. R. de Pouplana, "Role of tRNA modifications in human diseases," Trends in Molecular Medicine, vol. 20, no. 6, pp. 306-314, 2014.

[60] W. S. Yang and B. R. Stockwell, "Synthetic lethal screening identifies compounds activating iron-dependent, nonapoptotic cell death in oncogenic-RAS-harboring cancer cells," Chemistry \& Biology, vol. 15, no. 3, pp. 234-245, 2008.

[61] J. P. Friedmann Angeli, M. Schneider, B. Proneth et al., "Inactivation of the ferroptosis regulator $\mathrm{Gpx} 4$ triggers acute renal failure in mice," Nature Cell Biology, vol. 16, no. 12, pp. 11801191, 2014.

[62] A. P. Halestrap, E. Doran, J. P. Gillespie, and A. O'Toole, "Mitochondria and cell death," Biochemical Society Transactions, vol. 28, no. 2, pp. 170-177, 2000.

[63] F. N. Soria, A. Pérez-Samartín, A. Martin et al., "Extrasynaptic glutamate release through cystine/glutamate antiporter contributes to ischemic damage," The Journal of Clinical Investigation, vol. 124, no. 8, pp. 3645-3655, 2014.

[64] H. Tang, D. Chen, C. Li et al., "Dual GSH-exhausting sorafenib loaded manganese-silica nanodrugs for inducing 
the ferroptosis of hepatocellular carcinoma cells," International Journal of Pharmaceutics, vol. 572, p. 118782, 2019.

[65] S. Neitemeier, A. Jelinek, V. Laino et al., "BID links ferroptosis to mitochondrial cell death pathways," Redox Biology, vol. 12, pp. 558-570, 2017.

[66] H. Walczak, R. E. Miller, K. Ariail et al., “Tumoricidal activity of tumor necrosis factor-related apoptosis-inducing ligand in vivo," Nature Medicine, vol. 5, no. 2, pp. 157-163, 1999.

[67] A. M. Schmidt, "Highlighting diabetes mellitus: the epidemic continues," Arteriosclerosis, Thrombosis, and Vascular Biology, vol. 38, no. 1, pp. e1-e8, 2018.

[68] American Diabetes Association, "2. Classification and diagnosis of diabetes," Diabetes Care, vol. 38, Supplement_1, pp. S8-S16, 2015.

[69] N. Lascar, J. Brown, H. Pattison, A. H. Barnett, C. J. Bailey, and S. Bellary, "Type 2 diabetes in adolescents and young adults," The Lancet Diabetes and Endocrinology, vol. 6, no. 1, pp. 69-80, 2018.

[70] J. Wang and H. Wang, "Oxidative stress in pancreatic beta cell regeneration," Oxidative Medicine and Cellular Longevity, vol. 2017, Article ID 1930261, 9 pages, 2017.

[71] A. Bruni, A. R. Pepper, R. L. Pawlick et al., "Ferroptosisinducing agents compromise in vitro human islet viability and function," Cell Death \& Disease, vol. 9, no. 6, p. 595, 2018.

[72] M. Grau-Pérez, C. C. Kuo, M. Spratlen et al., "The association of arsenic exposure and metabolism with type 1 and type 2 diabetes in youth: the SEARCH Case-Control Study," Diabetes Care, vol. 40, no. 1, pp. 46-53, 2017.

[73] R. Beck, M. Styblo, and P. Sethupathy, "Arsenic exposure and type 2 diabetes: microRNAs as mechanistic links?," Current Diabetes Reports, vol. 17, no. 3, p. 18, 2017.

[74] S. Wei, T. Qiu, X. Yao et al., "Arsenic induces pancreatic dysfunction and ferroptosis via mitochondrial ROS-autophagylysosomal pathway," Journal of Hazardous Materials, vol. 384, p. 121390, 2020.

[75] T. Kose, M. Vera-Aviles, P. A. Sharp, and G. O. LatundeDada, "Curcumin and (-)- epigallocatechin-3-gallate protect murine MIN6 pancreatic beta-cells against iron toxicity and erastin-induced ferroptosis," Pharmaceuticals, vol. 12, no. 1, 2019.

[76] Y. Jiao, J. Wilkinson IV, E. C. Pietsch et al., "Iron chelation in the biological activity of curcumin," Free Radical Biology \& Medicine, vol. 40, no. 7, pp. 1152-1160, 2006.

[77] L. Sun, G. Zong, A. Pan et al., "Elevated plasma ferritin is associated with increased incidence of type 2 diabetes in middle-aged and elderly Chinese adults," The Journal of Nutrition, vol. 143, no. 9, pp. 1459-1465, 2013.

[78] D. Basuli, R. G. Stevens, F. M. Torti, and S. V. Torti, "Epidemiological associations between iron and cardiovascular disease and diabetes," Frontiers in Pharmacology, vol. 5, p. 117, 2014.

[79] J. Huang, D. Jones, B. Luo et al., "Iron overload and diabetes risk: a shift from glucose to fatty acid oxidation and increased hepatic glucose production in a mouse model of hereditary hemochromatosis," Diabetes, vol. 60, no. 1, pp. 80-87, 2011.

[80] M. Jehn, J. M. Clark, and E. Guallar, "Serum ferritin and risk of the metabolic syndrome in U.S. adults," Diabetes Care, vol. 27, no. 10, pp. 2422-2428, 2004.

[81] R. C. Cooksey, H. A. Jouihan, R. S. Ajioka et al., "Oxidative stress, beta-cell apoptosis, and decreased insulin secretory capacity in mouse models of hemochromatosis," Endocrinology, vol. 145, no. 11, pp. 5305-5312, 2004.

[82] H. F. Escobar-Morreale, M. Luque-Ramírez, F. ÁlvarezBlasco, J. I. Botella-Carretero, J. Sancho, and J. L. San Millán, "Body iron stores are increased in overweight and obese women with polycystic ovary syndrome," Diabetes Care, vol. 28, no. 8, pp. 2042-2044, 2005.

[83] S. N. Rajpathak, J. P. Crandall, J. Wylie-Rosett, G. C. Kabat, T. E. Rohan, and F. B. Hu, "The role of iron in type 2 diabetes in humans," Biochimica et Biophysica Acta, vol. 1790, no. 7, pp. 671-681, 2009.

[84] K. S. Houschyar, R. Lüdtke, G. J. Dobos et al., "Effects of phlebotomy-induced reduction of body iron stores on metabolic syndrome: results from a randomized clinical trial," BMC Medicine, vol. 10, no. 1, p. 54, 2012.

[85] J. M. Fernandez-Real, G. Penarroja, A. Castro, F. GarciaBragado, I. Hernandez-Aguado, and W. Ricart, "Blood letting in high-ferritin type 2 diabetes: effects on insulin sensitivity and beta-cell function," Diabetes, vol. 51, no. 4, pp. 10001004, 2002.

[86] W. Wang, M. A. Knovich, L. G. Coffman, F. M. Torti, and S. V. Torti, "Serum ferritin: past, present and future," Biochimica et Biophysica Acta, vol. 1800, no. 8, pp. 760-769, 2010.

[87] N. G. Forouhi, A. H. Harding, M. Allison et al., "Elevated serum ferritin levels predict new-onset type 2 diabetes: results from the EPIC-Norfolk prospective study," Diabetologia, vol. 50, no. 5, pp. 949-956, 2007.

[88] Z. Wang, H. B. Fan, W. W. Yang et al., "Correlation between plasma ferritin level and gestational diabetes mellitus and its impact on fetal macrosomia," Journal of diabetes investigation, vol. 9, no. 6, pp. 1354-1359, 2018.

[89] B. B. Yeap, M. L. Divitini, J. E. Gunton et al., "Higher ferritin levels, but not serum iron or transferrin saturation, are associated with type 2 diabetes mellitus in adult men and women free of genetic haemochromatosis," Clinical Endocrinology, vol. 82, no. 4, pp. 525-532, 2015.

[90] H. Elimam, A. M. Abdulla, and I. M. Taha, "Inflammatory markers and control of type 2 diabetes mellitus," Diabetes and Metabolic Syndrome: Clinical Research and Reviews, vol. 13, no. 1, pp. 800-804, 2019.

[91] D. Pain and A. Dancis, "Roles of Fe-S proteins: from cofactor synthesis to iron homeostasis to protein synthesis," Current Opinion in Genetics \& Development, vol. 38, pp. 45-51, 2016.

[92] F. Y. Wei, T. Suzuki, S. Watanabe et al., "Deficit of tRNA(Lys) modification by Cdkall causes the development of type 2 diabetes in mice," The Journal of Clinical Investigation, vol. 121, no. 9, pp. 3598-3608, 2011.

[93] F. Y. Wei and K. Tomizawa, "Functional loss of Cdkal1, a novel tRNA modification enzyme, causes the development of type 2 diabetes," Endocrine Journal, vol. 58, no. 10, pp. 819-825, 2011.

[94] N. P. Mena, P. J. Urrutia, F. Lourido, C. M. Carrasco, and M. T. Núñez, "Mitochondrial iron homeostasis and its dysfunctions in neurodegenerative disorders," Mitochondrion, vol. 21, pp. 92-105, 2015.

[95] K. Rehman and M. S. H. Akash, "Mechanism of generation of oxidative stress and pathophysiology of type 2 diabetes mellitus: how are they interlinked?," Journal of Cellular Biochemistry, vol. 118, no. 11, pp. 3577-3585, 2017.

[96] J. B. de Haan, C. Bladier, P. Griffiths et al., "Mice with a homozygous null mutation for the most abundant 
glutathione peroxidase, Gpx1, show increased susceptibility to the oxidative stress-inducing agents paraquat and hydrogen peroxide," The Journal of Biological Chemistry, vol. 273, no. 35, pp. 22528-22536, 1998.

[97] Y. S. Ho, J. L. Magnenat, R. T. Bronson et al., "Mice deficient in cellular glutathione peroxidase develop normally and show no increased sensitivity to hyperoxia," The Journal of Biological Chemistry, vol. 272, no. 26, pp. 1664416651, 1997.

[98] K. Loh, H. Deng, A. Fukushima et al., "Reactive oxygen species enhance insulin sensitivity," Cell Metabolism, vol. 10, no. 4, pp. 260-272, 2009.

[99] P. Newsholme, E. P. Haber, S. M. Hirabara et al., "Diabetes associated cell stress and dysfunction: role of mitochondrial and non-mitochondrial ROS production and activity," The Journal of Physiology, vol. 583, no. 1, pp. 9-24, 2007.

[100] E. A. Killion, A. R. Reeves, M. A. el Azzouny et al., "A role for long-chain acyl-CoA synthetase-4 (ACSL4) in diet-induced phospholipid remodeling and obesity-associated adipocyte dysfunction," Molecular metabolism, vol. 9, pp. 43-56, 2018.

[101] H. A. Israr-ul, M. J. Longacre, S. W. Stoker et al., "Characterization of Acyl-CoA synthetase isoforms in pancreatic beta cells: gene silencing shows participation of ACSL3 and ACSL4 in insulin secretion," Archives of Biochemistry and Biophysics, vol. 618, pp. 32-43, 2017.

[102] W. Li, W. Li, Y. Leng, Y. Xiong, and Z. Xia, "Ferroptosis is involved in diabetes myocardial ischemia/reperfusion injury through endoplasmic reticulum stress," DNA and Cell Biology, vol. 39, no. 2, pp. 210-225, 2020.

[103] W. H. Xu, C. H. Li, and T. L. Jiang, "Ferroptosis pathway and its intervention regulated by Chinese materia medica," Zhongguo Zhong Yao Za Zhi, vol. 43, no. 20, pp. 40194026, 2018.

[104] D. P. Del Re, D. Amgalan, A. Linkermann, Q. Liu, and R. N. Kitsis, "Fundamental mechanisms of regulated cell death and implications for heart disease," Physiological Reviews, vol. 99, no. 4, pp. 1765-1817, 2019.

[105] C. E. Ndumele, K. Matsushita, M. Lazo et al., "Obesity and subtypes of incident cardiovascular disease," Journal of the American Heart Association, vol. 5, no. 8, 2016.

[106] P. Valensi and C. Meune, "Congestive heart failure caused by silent ischemia and silent myocardial infarction : diagnostic challenge in type 2 diabetes," Herz, vol. 44, no. 3, pp. 210217, 2019.

[107] Y. Baba, J. K. Higa, B. K. Shimada et al., "Protective effects of the mechanistic target of rapamycin against excess iron and ferroptosis in cardiomyocytes," American Journal of Physiology. Heart and Circulatory Physiology, vol. 314, no. 3, pp. H659-H668, 2018.

[108] E. R. Dabkowski, C. L. Williamson, and J. M. Hollander, "Mitochondria-specific transgenic overexpression of phospholipid hydroperoxide glutathione peroxidase (GPx4) attenuates ischemia/reperfusion-associated cardiac dysfunction," Free Radical Biology \& Medicine, vol. 45, no. 6, pp. 855-865, 2008.

[109] W. S. Lee and J. Kim, "Diabetic cardiomyopathy: where we are and where we are going," The Korean Journal of Internal Medicine, vol. 32, no. 3, pp. 404-421, 2017.

[110] T. H. Marwick, R. Ritchie, J. E. Shaw, and D. Kaye, "Implications of underlying mechanisms for the recognition and management of diabetic cardiomyopathy," Journal of the
American College of Cardiology, vol. 71, no. 3, pp. 339-351, 2018.

[111] A. R. Thandavarayan, V. V. Giridharan, K. Watanabe, and T. Konishi, "Diabetic cardiomyopathy and oxidative stress: role of antioxidants," Cardiovascular \& Hematological Agents in Medicinal Chemistry, vol. 9, no. 4, pp. 225-230, 2011.

[112] Q. M. Chen and A. J. Maltagliati, "Nrf2 at the heart of oxidative stress and cardiac protection," Physiological Genomics, vol. 50, no. 2, pp. 77-97, 2018.

[113] Z. Fan, A. K. Wirth, D. Chen et al., "Nrf2-Keap1 pathway promotes cell proliferation and diminishes ferroptosis," Oncogene, vol. 6, no. 8, article e371, 2017.

[114] M. Abdalkader, R. Lampinen, K. M. Kanninen, T. M. Malm, and J. R. Liddell, "Targeting Nrf2 to suppress ferroptosis and mitochondrial dysfunction in neurodegeneration," Frontiers in Neuroscience, vol. 12, p. 466, 2018.

[115] X. Hu, M. Rajesh, J. Zhang et al., "Protection by dimethyl fumarate against diabetic cardiomyopathy in type 1 diabetic mice likely via activation of nuclear factor erythroid- 2 related factor 2," Toxicology Letters, vol. 287, pp. 131-141, 2018.

[116] G. Wang, X. Song, L. Zhao, Z. Li, and B. Liu, "Resveratrol prevents diabetic cardiomyopathy by increasing Nrf2 expression and transcriptional activity," BioMed Research International, vol. 2018, Article ID 2150218, 13 pages, 2018.

[117] A. Ganeshpurkar and A. K. Saluja, "The pharmacological potential of Rutin," Saudi pharmaceutical journal, vol. 25, no. 2, pp. 149-164, 2017.

[118] R. Huang, Z. Shi, L. Chen, Y. Zhang, J. Li, and Y. An, "Rutin alleviates diabetic cardiomyopathy and improves cardiac function in diabetic ApoEknockout mice," European Journal of Pharmacology, vol. 814, pp. 151-160, 2017.

[119] Z. D. Ge, Q. Lian, X. Mao, and Z. Xia, "Current status and challenges of NRF2 as a potential therapeutic target for diabetic cardiomyopathy," International Heart Journal, vol. 60, no. 3, pp. 512-520, 2019. 